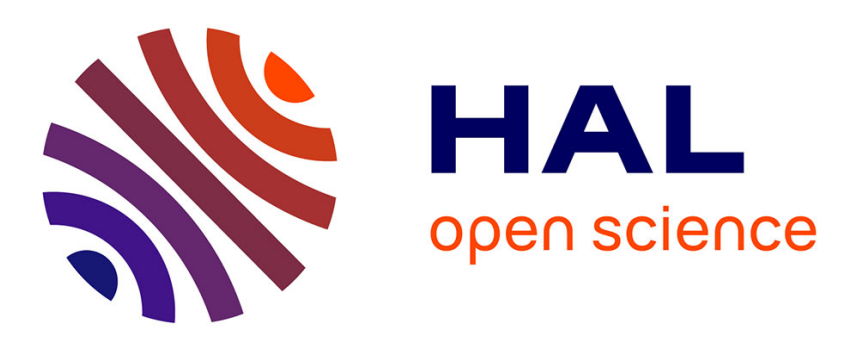

\title{
Critical behaviour in a field for uniaxial ferroelectrics and ferromagnets with dipolar interaction
}

\author{
C. Bervillier
}

\section{To cite this version:}

C. Bervillier. Critical behaviour in a field for uniaxial ferroelectrics and ferromagnets with dipolar interaction. Journal de Physique Lettres, 1975, 36 (10), pp.225-228. 10.1051/jphyslet:019750036010022500 . jpa-00231193

\section{HAL Id: jpa-00231193 https://hal.science/jpa-00231193}

Submitted on 1 Jan 1975

HAL is a multi-disciplinary open access archive for the deposit and dissemination of scientific research documents, whether they are published or not. The documents may come from teaching and research institutions in France or abroad, or from public or private research centers.
L'archive ouverte pluridisciplinaire HAL, est destinée au dépôt et à la diffusion de documents scientifiques de niveau recherche, publiés ou non, émanant des établissements d'enseignement et de recherche français ou étrangers, des laboratoires publics ou privés. 


\title{
CRITICAL BEHAVIOUR IN A FIELD FOR UNIAXIAL FERROELECTRICS AND FERROMAGNETS WITH DIPOLAR INTERACTION
}

\author{
C. BERVILLIER
}

\begin{abstract}
Service de Physique Théorique, Centre d'Etudes Nucléaires de Saclay
\end{abstract}
BP 2, 91190 Gif-sur-Yvette, France

(Reçu le 9 juin 1975, accepté le 30 juin 1975)

\begin{abstract}
Résumé. - L'objet de cet article est d'obtenir les singularités logarithmiques qui apparaissent dans les systèmes dipolaires uniaxes (ou bien dans la théorie à quatre dimensions) lorsque l'on approche du point critique alors que le champ extérieur $H$ n'est pas nul. Le résultat que l'on obtient ne prend une forme simple que pour $n=1, n$ étant le nombre de composantes du paramètre d'ordre. Par exemple l'inverse de la susceptibilité magnétique se comporte comme $\chi^{-1} \sim \chi_{\text {c.m. }}^{-1}\left|\log \chi_{\text {c.m. }}^{-1}\right|^{-1 / 3}$ où c.m. se réfère aux quantités calculées dans la théorie du champ moyen lorsque $H$ n'est pas nul.

Abstract. - The purpose of this paper is to obtain the logarithmic critical singularities for uniaxial dipolar systems (or for the four dimensional theory) in non zero external field. The result takes a simple form only for $n=1, n$ being the number of components of the order parameter. For example the inverse magnetic susceptibility behaves as $\chi^{-1} \sim \chi_{\text {m.f. }}^{-1}\left|\log \chi_{\text {m.f. }}^{-1}\right|^{-1 / 3}$ in which m.f. refers to mean field value when $H$ is non zero.
\end{abstract}

1. Introduction. - Several years ago, Larkin and Khmel'nitskii predicted that the critical behaviour of uniaxial ferroelectrics [1] or ferro-magnets [2] with strong dipolar interactions should be classical apart from logarithmic terms. This was recently confirmed experimentally by the measurements of the logarithmic contribution to the specific heat of the dipolar ferromagnet $\mathrm{LiTbF}_{4}$ by Ahlers et al. [3]. Recent measurements on ferroelectric substances, [4] the triglycine sulfates (T.G.S.) also revealed this classical behaviour and were compatible with the predicted power of the logarithm but with much less accuracy. Other measurements [5] were done on T.G.S. in a field, but there was no calculation available to make contact with theory. Of course, the renormalizationgroup analysis readily leads to an answer to this question. The simplest way of writing this answer in terms of $M$ and $t$ is :

$$
\chi_{(M, t)}^{-1} \sim \chi_{\mathrm{m} . \mathrm{f} .}^{-1}(\tilde{M}, t)\left[\log \left(\chi_{\mathrm{m} . \mathrm{f}}^{-1}(\tilde{M}, t)\right)\right]^{-1 / 3}
$$

for the magnetic susceptibility, and

$$
C(\tilde{M}, t) \sim C_{\text {m.f. }}(\tilde{M}, t)\left[\log \left(\chi_{\text {m.f. }}^{-1}(\tilde{M}, t)\right)\right]^{1 / 3}
$$

for the specific heat, in which the symbol m.f. refers to the mean field value near $T_{\mathrm{c}}$, i.e. :

$$
\chi_{\mathrm{m} . \mathrm{f} .}^{-1}(\tilde{M}, t)=t+\frac{1}{2} g \tilde{M}^{2}
$$

$$
\begin{aligned}
C_{\mathrm{m} . \mathrm{f} .}(\tilde{M}, t) & =1+\frac{g \tilde{M}^{2}}{\left(t+\frac{g \tilde{M}^{2}}{2}\right)^{3}} \times \\
& \text { and } \quad \\
\times & {\left[\left(t+\frac{g \tilde{M}^{2}}{2}\right)^{2}-2 t\left(t+\frac{g \tilde{M}^{2}}{6}\right)\right] } \\
\tilde{M} & \sim a M\left[\log \left(\chi_{\mathrm{m} . \mathrm{f} .}^{-1}(a M, t)\right)\right]^{-1 / 3}
\end{aligned}
$$

$a$ being a constant.

As will be shown later, in terms of $H$ and $t$, we have to replace $\tilde{M}$ in eq. (1), (2) by the positive solution of the equation of state in mean field theory, which apart from a factor $a$ is :

$$
\frac{H}{a}=t \tilde{M}+g \frac{\tilde{M}^{3}}{6} .
$$

At first sight these results may look like almost obvious consequences of the result of Larkin and Khmel'nitskii, but one should stress the fact that this simple form (1) and (2) is valid exclusively for $n=1$. For $n>1$, the result can only be written in parametric form.

2. Calculations. - We will now use the ferromagnetic language, in the four dimensional theory (which at leading order describes the uniaxial dipolar system). 
In the critical domain, we cannot use perturbation theory in the actual coupling constant $g$ of the four spin interaction, since the coefficients of that expansion become larger and larger as one approaches the critical point. But the Renormalization Group techniques $[6,7]$ lead to an effective coupling constant in the critical domain which is small, behaving as the inverse of the logarithm of the correlation length $\xi$, so that perturbation theory can be applied in power of this effective coupling constant. Indeed we know that $[7,8]$ for example the free energy $\Gamma(M, t, g)$ satisfies a renormalization equation whose solutions have the following property :

$$
\Gamma(M, t, g)=\lambda^{4} \Gamma\left[\frac{M(\lambda)}{\lambda}, \frac{t(\lambda)}{\lambda^{2}}, g(\lambda)\right]
$$

in which

$$
\begin{aligned}
\ln \lambda & =\int_{g}^{g(\lambda)} \frac{\mathrm{d} y}{\beta(y)} \\
t(\lambda) & =t \exp \left[-\int_{g}^{g(\lambda)} \frac{1}{\frac{v(y)}{\beta(y)}} \mathrm{d} y\right] \\
M(\lambda) & =M \exp \left[-\frac{1}{2} \int_{g}^{g(\lambda)} \frac{\eta(y)}{\beta(y)} \mathrm{d} y\right] .
\end{aligned}
$$

The functions $\beta(g), \eta(g), v(g)$ can be determined in perturbation theory [7]

$$
\begin{gathered}
\beta(g)=\frac{n+8}{6} g^{2}+O\left(g^{3}\right) \\
\frac{1}{v(g)}-2=-\frac{n+2}{6} g+O\left(g^{2}\right) \\
\eta(g)=\frac{n+2}{72} g^{2}+O\left(g^{3}\right) .
\end{gathered}
$$

If perturbation theory can be applied, then integration of eq. (8), (10) gives, at leading order :

$$
\begin{aligned}
g(\lambda) & =\frac{g}{1+\frac{n+8}{6} g|\log \lambda|} \\
t(\lambda) & =t\left(\frac{g(\lambda)}{g}\right)^{\frac{n+2}{n+8}} \\
M(\lambda) & =M \exp \left[g \frac{n+2}{24(n+8)}\right] \equiv a M .
\end{aligned}
$$

Now, the correlation length $\xi(M, t, g)$ is also a solution of a R.G.E. so it has the following property :

$$
\xi(M, t, g)=\lambda^{-1} \xi\left[\frac{M(\lambda)}{\lambda}, \frac{t(\lambda)}{\lambda^{2}}, g(\lambda)\right] .
$$

Until now $\lambda$ was an arbitrary parameter, but following K. G. Wilson [6] we can now choose it as the solution of

$$
\xi\left[\frac{M(\lambda)}{\lambda}, \frac{t(\lambda)}{\lambda^{2}}, g(\lambda)\right]=1
$$

for which $\lambda=[\xi(M, t, g)]^{-1}$.

So eq. (17) and (18) show that $\lambda$ goes to zero when $\xi(M, t, g)$ becomes infinite, that is to say as one approaches the critical point. Thus eq. (14) shows that $g(\lambda)$ goes to zero as $|\log \lambda|^{-1}$ while $\xi(\lambda)$ is finite and then perturbation theory may be applied.

Let us define the inverse square of the correlation length as the second moment of the spin-spin correlation function

$$
\xi_{1}^{-2}=\frac{\Gamma^{(2)}(p=0 ; t, M, g)}{\frac{\partial}{\partial p^{2}} \Gamma^{(2)}\left(p^{2} ; t, M, g\right) \mid p^{2}=0}
$$

$\Gamma^{(2)}\left(p^{2}, t, M, y\right)$ is the inverse of the spin-spin correlation function. In perturbation theory eq. (18) and (19) give :

$$
t(\lambda)+\frac{1}{2} g(\lambda) M^{2}(\lambda)=\lambda^{2} .
$$

We have to solve eq. (20) for $\lambda$, and when we want to express our results in terms of $H$ and $t$ ( $H$ is the external magnetic field), we replace $M^{2}$ by its expression in terms of $H$ and $t$ via the equation of state. In perturbation theory we have :

$$
H(M, t, g)=\frac{\partial}{\partial M} \Gamma(M, t, g)=t M+\frac{g M^{3}}{6}
$$

and the R.G.E. gives

$$
H(M, t, g)=\lambda^{3} \frac{M(\lambda)}{M} H\left[\frac{M(\lambda)}{\lambda}, \frac{t(\lambda)}{\lambda^{2}}, g(\lambda)\right] .
$$

Using eq. (16), (21) and (22), $M(\lambda)$ satisfies the following equation :

$$
M^{3}(\lambda)+6 \frac{t(\lambda)}{g(\lambda)} M(\lambda)-\frac{6 H}{a g(\lambda)}=0
$$

which has only one positive solution [9] for $H \neq 0$ :

$$
\text { a) } \begin{aligned}
M(\lambda)=\Phi\left[\frac{3 H}{a g(\lambda)} ; 2 \frac{t(\lambda)}{g(\lambda)}\right] \\
=\left[\frac{2}{g}\left(\frac{g(\lambda)}{g}\right)^{\frac{n+2}{n+8}-1}\right]^{1 / 2} \times \\
\left.\times \Phi\left(H^{\prime}\left(\frac{g(\lambda)}{g}\right)^{\frac{1-n}{n+8}} ; t\right)\right\}
\end{aligned}
$$

with 
So using eq. (11), $\lambda$ satisfies the following equation :

$\lambda^{2}=\left(\frac{g(\lambda)}{g}\right)^{\frac{n+2}{n+8}} f\left[H^{\prime}\left(\frac{g(\lambda)}{g}\right)^{\frac{1-n}{n+8}} ; t\right]$

(a)

in which

$$
f(x ; y)=[\Phi(x ; y)]^{2}+y
$$

As we approach the critical point, $\lambda$ goes to zero and $g(\lambda)$ becomes small going as eq. (14) :

$$
g(\lambda) \sim \frac{6}{n+8}|\log \lambda|^{-1}
$$

So eq. (25) leads to :

$$
\begin{array}{r}
\log \lambda=\frac{1}{2} \log \left\{f\left[H^{\prime}\left(\frac{g(\lambda)}{g}\right)^{\frac{1-n}{n+8}} ; t\right]\right\}+ \\
+\log \log \text { terms }
\end{array}
$$

and, for $n=1$, has one uniform solution in $H$ and $t$ :

$$
\log \lambda=\frac{1}{2} \log f\left(H^{\prime}, t\right)+\log \log \text { terms . }
$$

The magnetic susceptibility is, in perturbation theory :

$$
\chi^{-1}=t(\lambda)+\frac{1}{2} g(\lambda) M^{2}(\lambda)
$$

and, in the critical domain, for $n=1$ :

$$
\chi^{-1}(H, t) \propto\left[\frac{1}{1+\frac{3 g}{4} \log \left|f\left(H^{\prime}, t\right)\right|}\right]^{1 / 3} f\left(H^{\prime}, t\right) \text {. }
$$

In fact $f\left(H^{\prime}, t\right)$ is the inverse susceptibility, in terms of $H$ and $t$, calculated in mean field theory with a rescaled field $H / a$, thus one sees that, close to critical point, the behaviour of $\chi^{-1}$ is no longer described by mean field theory :

$$
\chi^{-1}(H, t) \propto \chi_{\text {m.f. }}^{-1}(H, t)\left[\log \left(\chi_{\text {m.f. }}^{-1}(H, t)\right)\right]^{-1 / 3} .
$$

In terms of $M$ and $t$, we have the same form, by (24a), (25b), (27) and (29)

$$
\chi^{-1}(M, t) \propto \chi_{\text {m.f. }}^{-1}(\tilde{M}, t)\left[\log \left(\chi_{\text {m.f. }}^{-1}(\tilde{M}, t)\right)\right]^{-1 / 3}
$$

in which the magnetization does not have its actual value $M$ in the present approximation, but its mean field value $\tilde{M}$. Eq. (24a) exhibits the relation between these two values for $n=1$ :

$$
M \sim \frac{1}{a} \tilde{M}\left[\log \left(\chi_{\text {m.f. }}^{-1}(\tilde{M}, t)\right)\right]^{1 / 3}
$$

with

$$
\chi_{\mathrm{m} . \mathrm{f} .}^{-1}(\tilde{M}, t)=t+g \frac{\tilde{M}^{2}}{2}
$$

And at leading order :

$$
\tilde{M} \simeq a M\left[\log \chi_{\text {m.f. }}^{-1}(a M, t)\right]^{-1 / 3} .
$$

It follows that

$$
\begin{aligned}
\chi^{-1}(M, t) \simeq \frac{t}{\left[\log \left(\chi_{\text {m.f. }}^{-1}(a M, t)\right)\right]^{1 / 3}}+ \\
\quad+\frac{g(a M)^{2}}{2 \log \left(\chi_{\text {m.f. }}^{-1}(a M, t)\right)}
\end{aligned}
$$

The specific heat is proportional to the negative of the second derivative with respect to $t$ of the free energy for a constant external field.

For $n=1$, perturbation theory gives :

$$
\Gamma_{\text {pert. th. }}(M, t, g)=\frac{t M^{2}}{2}+\frac{g M^{4}}{24}-\frac{t^{2}}{2 g} .
$$

The last term of the r.h.s. of eq. (36) is due to the additive renormalization of the energy-energy correlation function.

In the critical domain, we can use eq. (36) :

$$
\Gamma(M, t, g) \simeq \frac{t(\lambda) M^{2}(\lambda)}{2}+g(\lambda) \frac{M^{4}(\lambda)}{24}-\frac{t^{2}(\lambda)}{2 g(\lambda)}
$$

And so, using eq. (24) and (26)

$$
\left.\begin{array}{l}
\Gamma(M, t, g) \sim \frac{1}{g}\left[\frac{1}{1+\frac{3}{4} g \log \left(\chi_{\text {m.f. }}^{-1}(H, t)\right)}\right]^{-1 / 3} \times \\
\quad \times\left[-\frac{4}{3} t^{2}+\frac{2}{3} t \chi_{\text {m.f. }}^{-1}(H, t)+\frac{1}{6} \chi_{\text {m.f. }}^{-2}(H, t)\right]
\end{array}\right\} .
$$

Then one finds the following expression for the specific heat :

$$
C \propto C_{\text {m.f. }}\left[\frac{1}{1+\frac{3 g}{4} \log \chi_{\text {m.f. }}^{-1}}\right]^{-1 / 3}
$$

in which $C_{\text {m.f. }}$ is calculated in mean field theory, that is to say :

$$
\begin{aligned}
C_{\text {m.f. }}=1 & +\frac{g \tilde{M}^{2}}{\left(t+\frac{g \tilde{M}^{2}}{2}\right)^{3}} \times \\
& \times\left[\left(t+\frac{g \tilde{M}^{2}}{2}\right)^{2}-2 t\left(t+\frac{g \tilde{M}^{2}}{6}\right)\right]
\end{aligned}
$$

and in which $\tilde{M}$ is the mean field value of the magnetization related to $M$ by eq. (34).

Acknowledgments. - I would like to thank Dr E. Brezin who suggested to me the subject of this paper and with whom I had fruitfull discussions. 


\section{References}

[1] LARKin, A. I. and KhmeL'NITSKII, D. E., Zh. Eksp. Teor. Fiz. 56, 2087 (Soviet Physics JETP 29 (1969) 1123).

[2] Aharony, A., Phys. Lett. A 44 (1973) 313; Phys. Rev. B 8 (1973) 3363.

[3] Ahlers, G., Kornblit, A. and Guggenheim, H., to be published in Phys. Rev. Lett.

[4] Schürmann, H. K., Gillespie, S., Gunton, J. D. and MihaliSIN, T., Phys. Lett. A 45 (1973) 417.

[5] Gillespie, S., SchürmanN, H. K., Gunton, J. D. and Mihalisin, T., Solid State Commun. 15 (1974) 1753.

[6] Wilson, K. G. and Kogut, J., Phys. Rep. 12C (1974).
[7] Brézin, E., Le Guillou, J. C. and Zinn-Justin, J., Field theoretical approach to critical phenomena Saclay preprint DPh-T/74/100 to be published in Vol VI of Phase Transitions and Critical Phenomena (Academic Press) C. Domb and M. S. Green.

[8] Brézin, E., Le Guillou, J. C. and Zinn-Justin, J., Phys. Rev. D 8 (1973) 2418.

Brézin, E., J. Physique Lett. 36 (1975) L51.

[9] In order to find this positive solution we have cut the complex plane on the negative part of the real axis. 